\title{
La importancia de la formación inicial docente y su gestión para el acceso al mundo laboral
}

Enviado: 15 de mayo de 2020 / Aceptado: 26 de septiembre de 2020

Publicado: 30 de diciembre de 2020

MEDINA LÓPEZ, $M^{\mathrm{a}}$ ISABEL

Universidad de Granada, España

mimedinalopez@correo.ugr.es

NAVARRO JURADO, M MESÚS

Universidad de Granada, España

mariajesusnj@correo.ugr.es

ALONSO GARCÍA, SANTIAGo

Universidad de Granada, España

salonsog@ugr.es

DOI 10.24310/IJNE3.2.2020.9034

\section{RESUMEN}

A día de hoy, como futuras docentes, nos hacemos preguntas sobre la formación con la que, en un futuro, entraremos en nuestros puestos de trabajo y si esta será suficiente para transmitir un correcto aprendizaje a nuestros alumnos.

A partir de la consulta de la posición de España en los informes PISA, consideramos relevante un estudio acerca de la formación y calidad de la selección del profesorado de nuevo acceso en nuestro país así como los objetivos educativos, ya que, a partir de esta escasa formación y correcta administración de los futuros profesores, esta profesión se ha convertido en una de las menos valoradas en nuestro país, aun siendo de las más importantes para nuestra sociedad.

Partiendo de estos puntos flacos, hemos considerado algunos métodos y propuestas que, a nuestro parecer,

\section{ABSTRACT}

The importance of the initial teacher training and its process for the access to the professional work

Nowadays, as future teachers, we wonder about the training which we will get our job in the future and if this will be enough to teach a correct education for our students. From Spain's enquiry position in reports' PISA, we consider relevant as a study about the formation and quality of the selection of new teachers in our country as the educational objectives, since this shortage education and correct administration of the future teachers has become one of the less valued in our country even though being of the most important for our society.

Starting from these weaknesses, we have considered some methods and propose that, in our opinion, will improve our teacher education programs. After rea- 
mejorarían nuestros programas de formación docente. Tras la lectura de diversos artículos y la consulta de numerosas investigaciones hemos llegado a la redacción de las posibles soluciones que consideramos eficaces para alcanzar una calidad educativa en un futuro e intentar conseguir en España un mayor respeto para esta profesión.

Palabras Clave: calidad educativa, educación, programas de formación docente, objetivos educativos. ding some articles and consulting many investigations we have written some possible solutions that we consider effective to get an educational quality in a future and achieving a major respect for this profession in Spain.

Keywords: educational quality, education, teacher education programs, educational objectives.

\section{INTRODUCCIÓN}

Atendiendo a la definición de docencia de María Estela Raffino "profesión cuyo objetivo principal es transmitir la enseñanza a otras personas", nos gustaría hacer frente y comentar la actual formación que se recibe para ejercer esta profesión y cuestionarnos si realmente es eficaz en nuestro país.

Para comprobar la efectividad que la educación tiene en nuestro país, el primer estudio a consultar debería ser el informe PISA (Programme for International Student Assessment), en el que se evalúan los conocimientos adquiridos por los alumnos en un examen específico realizado al terminar la etapa de secundaria en más de 80 países del mundo. A partir de esto, se elabora una lista con los resultados adquiridas, ofreciendo así un balance del nivel educativo ofrecido en cada uno de estos países (Ministerio de Educación y Formación Profesional, 2019).

Tras analizar las calificaciones de los últimos informes realizados en 2018, España se encuentra en el puesto 28 en el ámbito de matemáticas y en el 25 en el de ciencias con unas medias de 481 y 483 respectivamente. Los alumnos españoles continúan por debajo de la media OCDE y además empeoran los resultados con respecto al informe de 2015 (Ministerio de Educación y Formación Profesional, 2019). Si observamos y analizamos estos resultados, nos damos cuenta de que la educación española está fallando en algunos aspectos y aún nos queda mucho por mejorar.

Uno de los aspectos más influyentes en la educación y por lo tanto en los resultados de los alumnos, es el profesorado. Éste, es el encargado de proporcionar los conocimientos de las diferentes materias y asegurar la adquisición de los mismos de una manera significativa. Consideramos oportuno el análisis de la formación y la motivación que estos tienen durante 
sus estudios universitarios para observar la calidad de los mismos y por lo tanto el efecto que esto tendrá en su futura labor como docentes. Investigar en relación a la propuesta del MIR a nivel educativo que se ideó hace unos años por nuestro gobierno y que a día de hoy sigue sin considerarse a nivel legal y observar otros aspectos, disciplinas y procedimientos llevados a cabo en otros países partícipes en los informes PISA con resultados más altos, son los aspectos que nos gustaría tratar para proporcionar soluciones a la falta de efectividad que tiene el sistema educativo vigente en nuestro país.

Teniendo a los docentes como uno de los pilares de la educación y principales agentes de cambio en una sociedad, ¿se les da el valor que verdaderamente tienen?¿Están lo suficientemente formados para hacer frente a tal responsabilidad? ¿Son suficientes los exámenes de acceso a la universidad? ¿Se les hace conscientes de la importancia de su profesión al ingresar en sus estudios de grado? ¿Son formados en todas las áreas necesarias para ofrecer un aprendizaje significativo? ¿Se debería de limitar la oferta de plazas en grados como Educación Primaria?

Nuestra preocupación por la actual formación en la educación española es la que nos ha llevado a realizar este proyecto. Resolver estas cuestiones e intentar proponer una mejora a este sistema, serán los objetivos principales, atendiendo siempre a las formaciones extranjeras que favorezcan a la propia.

A día de hoy, nos podemos dar cuenta que la educación es una de las profesiones menos valoradas en España. Por ello, la docencia española se ha tenido que ver respaldada a efectos legales para conseguir la autoridad y el respeto merecidos haciéndose vigente con la Ley de Autoridad del Profesor de la Comunidad de Madrid o Ley de Autoridad Docente de la Región de Murcia entre otras (Rebolledo Gámez, 2015, p. 130). Es cada vez más normal presenciar momentos en los que los padres se consideran con mayor formación para la guía de la educación de sus hijos que los profesores, es por esto que se menosprecia tanto este grado. Esto se ha visto reforzado por el hecho de que el acceso a la Universidad en nuestro país se vea controlado por la realización de un examen general, tras el cual, si se obtiene una nota media adecuada, se podrá entrar a cualquier carrera ofrecida por las universidades (Zarazaga, J. M. E.,2006, p.29). Esto hace que, muchas de las personas que accedan al grado en educación lo hagan de rebote o porque no tengan ninguna otra opción tras no haber entrado en sus elecciones preferentes (Rebolledo Gámez, 2015, p. 137), a posteriori esto se verá reflejado en su futuro profesional cuando ejerzan como profesores sin querer estar ahí, sólo por ganar un sueldo.

El gran número de docentes que se encuentra en esta situación, ejerciendo sin actitud ni voluntad, hace que esta profesión se menosprecie cada día más y se le tenga menos respeto. 
La estructura en la formación, al igual que el acceso a las universidades, juega un papel muy importante en la toma de valor de muchas profesiones, por ejemplo, la medicina es una profesión muy bien valorada debido a los largos años de estudios que ésta conlleva y se tiene un gran respeto por la misma debido al sacrificio y valor que los médicos deben tener. Sin embargo en la formación educativa concebimos dos modelos: el concurrente y el consecutivo en función de la división o no de la disciplina y la didáctica, de los que ninguno de los dos superaría los 5 años de estudios ni tendría una gran práctica en el mundo laboral de forma tutelada (Ministerio de Educación y Formación Profesional, 2019, pp.12-13).

En el caso de España observamos que se llevan a cabo los dos métodos en función del nivel para el que estés estudiando. En el ámbito de educación secundaria se da un método consecutivo, ya que, para ejercer como profesor en un instituto, debes formarte previamente en una materia específica para después realizar un máster de especificación curricular tras el cual puedes acceder al mercado laboral. La carencia de formación focalizada a la docencia será aún mayor, ya que a lo largo de la especialidad no adquirirán ningún conocimiento sobre cómo poder transmitir lo que saben (Rebolledo Gámez, 2015, p. 134) y esto sólo se les facilitará a lo largo de un año de máster que, a nuestro parecer, es escaso. Por otro lado, en los estudios de educación primaria se observa un método simultáneo en el que se van adquiriendo los conocimientos a la vez que su didáctica, por lo que se entiende que acabados los 4 años de grado ya se puede ejercer. A lo largo de sus estudios las personas matriculadas en el grado de educación primaria cursarán una serie de materias para formarse orientados a la integración de contenidos científicos, didácticos y profesionales, pero no debe dejarse de lado los problemas que se dan ante la falta de conexión entre la didáctica y el conocimiento, ya que la primera se encuentra aislada y no se percibe ninguna conexión aparente con el resto de aspectos. Se debe de resaltar además, la falta de horas prácticas a lo largo del curso de los años, ya que solo se llevará a cabo entre 3 y 4 meses en los dos últimos años de estudios. Esto hace ver una falta de contacto con el ámbito laboral y una falta de experiencia que obstaculiza la observación de actitudes y aptitudes que cada persona tiene en su profesión a la hora de la elección docente (Rebolledo Gámez, 2015, p. 134). A lo largo de los estudios de grado, tras la implantación de la EEES, los alumnos deberás de haber adquirido las siguientes competencias (Ministerio de Educación y Formación Profesional, 2019, pp.13-14) que quedan reflejadas en el BOE $\mathrm{n}^{\circ} 312$, 2007, p. 53747- 53748:

\footnotetext{
"Conocer las áreas curriculares de la Educación Primaria, la relación interdisciplinar entre ellas, los criterios de evaluación y el cuerpo de conocimientos didácticos en torno a los procedimientos de enseñanza y aprendizaje respectivos.”
} 
“Diseñar, planificar y evaluar procesos de enseñanza y aprendizaje, tanto individualmente como en colaboración con otros docentes y profesionales del centro."

"Abordar con eficacia situaciones de aprendizaje de lenguas en contextos multiculturales y plurilingües. Fomentar la lectura y el comentario crítico de textos de los diversos dominios científicos y culturales contenidos en el currículo escolar."

"Diseñar y regular espacios de aprendizaje en contextos de diversidad y que atiendan a la igualdad de género, a la equidad y al respeto a los derechos humanos que conformen los valores de la formación ciudadana."

"Fomentar la convivencia en el aula y fuera de ella, resolver problemas de disciplina y contribuir a la resolución pacífica de conflictos. Estimular y valorar el esfuerzo, la constancia y la disciplina personal en los estudiantes."

“Conocer la organización de los colegios de Educación Primaria y la diversidad de acciones que comprende su funcionamiento. Desempeñar las funciones de tutoría y de orientación con los estudiantes y sus familias, atendiendo las singulares necesidades educativas de los estudiantes. Asumir que el ejercicio de la función docente ha de ir perfeccionándose y adaptándose a los cambios científicos, pedagógicos y sociales a lo largo de la vida”.

"Colaborar con los distintos sectores de la comunidad educativa y del entorno social. Asumir la dimensión educadora de la función docente y fomentar la educación democrática para una ciudadanía activa."

"Mantener una relación crítica y autónoma respecto de los saberes, los valores y las instituciones sociales públicas y privadas.”

"Valorar la responsabilidad individual y colectiva en la consecución de un futuro sostenible."

"Reflexionar sobre las prácticas de aula para innovar y mejorar la labor docente. Adquirir hábitos y destrezas para el aprendizaje autónomo y cooperativo y promoverlo entre los estudiantes.”

"Conocer y aplicar en las aulas las tecnologías de la información y de la comunicación. Discernir selectivamente la información audiovisual que contribuya a los aprendizajes, a la formación cívica y a la riqueza cultural."

"Comprender la función, las posibilidades y los límites de la educación en la sociedad actual y las competencias fundamentales que afectan a los colegios de Educación Primaria y a sus profesionales. Conocer modelos de mejora de la calidad con aplicación a los centros educativos.” 
Sin embargo, si bien están claramente estipuladas las capacidades a adquirir, el gran número de futuros docentes presentes en las aulas de las diversas facultades españolas dificulta la adquisición de todas ellas al conjunto del estudiantado e incluso la posterior selección en un concurso de los mejores profesores.

Con esta investigación queremos hacer ver la cantidad de métodos utilizados en los diversos países que participan en los programas PISA. Informar sobre la posibilidad de un MIR en educación para limitar el número de plazas y proporcionar unas prácticas de calidad docente. Cuestionar la formación docente en las TIC como método de actualización de la educación.

\section{MATERIAL Y MÉTODO}

La metodología empleada en esta investigación es bastante sencilla, pero extensa en su proceso ya que es un tema que abarca, a su vez, una gran extensión de subtemas. Hemos procedido a una recopilación cualitativa de los datos en la que hemos analizado una gran cantidad de fuentes primarias, como la investigación llevada a cabo por Francisco López Rupérez, que abarca los porqués del MIR educativo u otros estudios publicados en algunas de las diferentes revistas de la Universidad de Granada, la Universidad de Jaén o la Universidad de La Rioja.

Tras un largo tiempo de lectura y recopilación de información procedimos a reflejar los datos obtenidos de forma estructurada para poder proporcionar la información a continuación descrita como posibles soluciones que dar a nuestro problema introducido.

\section{RESULTADOS}

\subsection{MIR educativo}

Tras la investigación y lectura de algunas publicaciones del Dr.Francisco López Rupérez, el cual ha sido el único que hemos encontrado que haya tratado este tema, descubrimos que el MIR educativo propuesto para la formación docente en España en 2011 podría considerarse un pilar en esta tarea universitaria para realizar una modernización de nuestro sistema educativo.

Se utilizará como una herramienta para actuar sobre los requerimientos actuales en la profesión docente. 
Este término, según nos informa F. Rupérez en su informe de 2018, ha ido adquiriendo aceptación en la última década de este siglo y se considera a día de hoy uno de los instrumentos más poderoso en el cambio de la formación inicial del profesorado.

Pretende proporcionar docentes que al final de sus estudios sepan atender a la diversidad del alumnado atendiendo a las diferentes culturas, ritmos y estilos de aprendizaje (Rupérez, 2015).

Hace un enfoque en el único factor de cambio de la educación, los profesores, puesto que es su formación la única que puede ser modificada legalmente para la mejora del rendimiento académico. Para llegar a esto, se debe concretar un nuevo sistema para el acceso a la profesión eficaz, crear una "criba" que permita reconocer y terminar de preparar a los alumnos que tengan las características y el interés de participar activamente en el cambio y mejora de la educación (Rupérez, 2018).

La propuesta del MIR ofrece la solución a esta propuesta con la realización de un examen a nivel nacional en el que se seleccionen a aquellos alumnos que lo superen con las más altas calificaciones y opten a plaza gracias a ella. El número de plazas, será determinado, al igual que en medicina, por las plazas requeridas por el estado para la función docente, acabando así con la sobreabundancia que amenaza a la calidad formativa (Rupérez, 2015).

Su formación inicial se conseguiría actualizar a las necesidades actuales de la sociedad fomentando el trabajo en equipo entre profesores y el uso aplicado de la información, conocimientos y recursos informáticos adquiridos (Rupérez, 2018).

La finalidad es la de formar docentes especialistas que adquieran una enseñanza de calidad y que los prepare como grandes profesionales, desarrollando todas y cada una de las competencias para su futuro laboral. Para asegurarse del cumplimiento de esta función, serán evaluados a nivel formativo, anual y final, para de esta forma se logra hacer un seguimiento de los aspectos adquiridos y de aquellos a seguir trabajando (Rupérez, 2015).

Estos procesos que hacen más completa la profesión favorecen el incremento de reconocimiento social, por lo que el MIR generaría un estado de mayor confianza en el profesorado tras la realización y estudio de todos los niveles hasta llegar a ejercer la labor (Rupérez, 2018). Además se consigue un ámbito de igualdad formativa a nivel nacional que terminaría con los dispares resultados en los controles internacionales y que llevaría a la profesión docente hacia la excelencia (Rupérez, 2015). 


\subsection{La formación en las TIC}

El cambio social que ha ocasionado el aumento de carácter tanto cualitativo como cuantitativo de las TIC provoca un cambio en el nivel y preparación de la formación educativa. Por ello se debe educar a los niños en estos niveles para que aprendan a usarlos e interpretarlos desde el inicio de su aprendizaje (Aznar Díaz, Hinojo Lucena, \& Fernández Martín, 2002, pp. 253-254). Esta globalización y continua aparición de nuevos recursos y avances científicos y tecnológicos hace que la Universidad se vea inmersa en una sociedad de cambio (Alonso \& Alonso, 2014, p. 133)

A lo largo de su preparación, los futuros docentes no cursan ninguna asignatura obligatoria que les prepare para el uso de las TIC en el aula. Simplemente se contempla en el grado una mención en Informática con 5 asignaturas que se cursan a lo largo del tercer y cuarto año. (Tárraga Mínguez, R., Sanz Cervera, P., Pastor Cerezuela, G. \& Fernández Andrés, M.I., 2017, p.114). No obstante, el alumnado se mantiene cerca de las tecnologías en el transcurso del resto de materias dándoles un uso práctico a algunas de estas nuevas herramientas que favorecen a sus estudios. Es por esto que en un futuro la Universidad deberá incluir en sus planes educativos nuevas asignaturas o enfoques educativos que incluyan a estas TIC. Esto proporcionará al alumnado unas nuevas capacidades socio-económicas, analíticas, críticas... dentro de este campo que los beneficiará a la hora de construir sus propios conocimientos críticos y válidos (Alonso \& Alonso, 2014, p134).

Se puede decir que, en cierta forma, los educandos tienen el interés de aprender a integrar las nuevas tecnologías en su currículum. Su uso proporcionará un soporte y ayuda para sus futuros alumnos (Aznar Díaz, Hinojo Lucena, \& Fernández Martín, 2002, p. 255) ya que suponen un medio de investigación y facilitan diversas ayudas para visualizar o presentar los contenidos de múltiples formas. Además, sirven de motivación para aquellos que tienen dificultades en el aprendizaje, tienen algún problema de comunicación o sufren alguna discapacidad física (Marín \& Romero, 2009, pp. 100-101).

A pesar de este uso particular de las tecnologías, la oferta de una formación práctica sobre su uso es necesaria para dar a conocer la utilidad en el desarrollo de un currículo y los diferentes medios en los que se pueden encontrar y utilizar para su uso y proyección de estos en el aula (Marín \& Romero, 2009, p. 99).

Sin embargo, esta falta de formación para profesorado y su forma tradicional de desenvolverse en la escuela por gran parte de ellos (Aznar Díaz, Hinojo Lucena, \& Fernández Martín, 2002 , p. 255), hace que aparezcan problemas y dificultades por parte de los mismos debido a la 
aparición de inseguridades en los docentes a la hora de usarlas en clase y a la dificultad para incluirlas en sus currículos (Marín \& Romero, 2009, p. 102).

El sometimiento a un cambio por parte de la sociedad hace que simultáneamente la escuela deba adaptarse y por consiguiente añadir nuevas formaciones o reformar las ya existentes para que la enseñanza de estos cambios no suponga un problema o dificultad al docente. A la hora de la Adaptación a las TIC, la falta de presupuesto para invertir en estas y la limitada formación que todavía a día de hoy se ofrece a los futuros profesores dificultan esta adaptación a la sociedad (Aznar Díaz, Hinojo Lucena, \& Fernández Martín, 2002, p. 256).

Para poder fomentar el uso de las mismas y que resulte efectiva su formación se debería promover el método de aprendizaje "learning by doing" dejando a un lado el "teaching by telling" de esta forma se promueve el acercamiento a las prácticas de lo aprendido para que no se quede en una simple memorización del contenido a aprender (Marín \& Romero, 2009, p. 102).

\subsection{Métodos de mejora para la docencia}

Como hemos podido comprobar en la lectura de los informes PISA, Finlandia cuenta con uno de los puestos más altos en el informe a día de hoy. Si analizamos las diferencias con respecto a España en relación a la formación docente, podemos observar que estas son significativas.

Para obtener una plaza en la formación docente española es necesario realizar la EBAU (Evaluación de Bachillerato para el Acceso a la Universidad). La condición es obtener la nota media de la titulación, la cual se fija por las notas de las personas que solicitan el grado. (Rebolledo Gámez, 2015, p. 137) La poca efectividad de este medio de acceso, como hemos citado anteriormente, recae en la facilidad de que accedan al grado personas que realmente no quieran cursar este, pero no hayan tenido la posibilidad de obtener plaza en el grado que verdaderamente querían. Este hecho provoca que existan docentes españoles con poca motivación por su trabajo, lo que repercute instantáneamente en el resultado académico de sus alumnos.

Por otro lado, podemos investigar y analizar el acceso finlandés. El método cambia de manera radical. Una vez finalizada la Educación Secundaria, los alumnos deben realizar un examen de matriculación, pero esto no es todo, posteriormente deben ejecutar un examen a nivel nacional, VAKAVA. Dependiendo de la nota obtenida en esa prueba, los alumnos podrán elegir en qué universidad quieren cursar sus estudios. Posteriormente, las Universidades de Finlandia cuentan con la autonomía de crear unas pruebas específicas de acceso a la misma, es por ello que, normalmente, realizan una entrevista individual a cada candidato. En ella, se 
valorará tanto el perfil del estudiante como sus habilidades, conocimientos e intereses en el ámbito educativo. (Pérez Granados, 2014, p.114-115). Este método provoca que los alumnos tengan un mayor grado de competitividad y permite verificar la motivación e interés de los futuros docentes, garantizando así un profesorado de calidad. Cabe destacar la necesidad de tener unos requisitos básicos entre los que destacan el conocimiento de dos lenguas a parte de la materna y unas bases y dotes artísticas y musicales. Además, una vez hayan ingresado en la universidad las clases no contarán con más de 15 alumnos para de esta forma fomentar la investigación y la autoevaluación, despertando así una consciencia meta-cognitiva (García Perales \& Martín Sánchez, 2012, p. 79-81).

Por otro lado, contemplamos en Finlandia unos principios en los que se basa su educación entre los que cabe destacar el hecho de un sistema curricular nacional, en el que se establecen unas bases y valores iguales para todos los alumnos. A partir de ellos cada localidad o entidad podrá organizar e implementar su docencia de manera totalmente libre, adaptándose a su personalidad y a la de sus alumnos.

Además, cabe destacar la formación continua recibida en este país y el enfoque de investigación que se promueve. A lo largo de sus años de docentes, el profesorado continúa una formación a través de la cual consiguen unos conocimientos actualizados. A su vez, estos realizan tareas de investigación dentro de su ámbito y promueven un aprendizaje en la equidad ya que se les ofrece a todos los niños del país las mismas oportunidades para aprender y estudiar (Niemu, H., 2015, pp.390-393). Esta formación continua es ofrecida por el Estado finlandés proporcionando las diferentes posibilidades además de horas de excedencia para poder asistir a estos cursos (García Perales \& Martín Sánchez, 2012, p. 82).

La gran importancia que se le ha dado a la formación docente y al respeto de esta profesión promueven el respeto y confianza en los profesores de forma que los alumnos no sólo recurren a ellos en una relación enseñanza-aprendizaje, sino que además les plantean problemas que les puedan suceder (García Perales \& Martín Sánchez, 2012, p. 82).

\section{CONCLUSIÓN}

Para terminar, nos gustaría destacar que con esta investigación hemos tomado conciencia de la gran problemática que tiene España en relación a la formación de docentes, hecho al que no se le da la importancia que requiere. Formar a los pequeños no solo en contenido básico sino también en valores es la finalidad de esta profesión, ya que, posteriormente, serán ellos los encargados de seguir evolucionando para conseguir una mejor sociedad. 
La formación docente en España cuenta con diversos puntos débiles. Tratar de darles una solución en un futuro cercano a través de diferentes propuestas ya existentes, el aumento en algunos de los aspectos de más actualidad como pueden ser las TIC o la observación e incluso mejora de algunos países con una mejor formación, podría hacer que tanto la carrera en particular como la educación en general adquieran la importancia que verdaderamente tienen para nuestra sociedad.

Uno de los aspectos que más nos han llamado la atención es la existencia de una propuesta de MIR educativo. Esto consiste en un examen a nivel nacional tras la realización del máster de educación para acceder a una formación práctica tutelada similar a la que realizan los médicos en su especialidad. Con esta propuesta se resalta la importancia que tienen las prácticas en esta profesión, ya que es una vez en el aula cuando de verdad estás ejerciendo y aplicando los contenidos que durante 5 años has aprendido. Esta propuesta no acogería al total de alumnos salientes de su formación universitaria sino que, tras la evaluación realizada entrarían las notas más altas hasta completar las plazas ofrecidas, número que variará en función de la necesidad nacional para completar la plantilla docente de la totalidad de colegios.

Como bien hemos dicho, la formación en las TIC de cara a una inclusión de las mismas en el currículo docente es escasa. La importancia de las nuevas tecnologías de la comunicación y la información se ha manifestado este año 2020. La actual crisis sanitaria que estamos viviendo en nuestro país ha sacado a la luz uno de los vacíos que nuestros docentes tienen en su formación. Al ser Internet la única forma de seguir con el normal transcurso del año escolar, nos hemos dado cuenta de que muchos de los profesores que actualmente están ejerciendo, carecen de una correcta formación en los recursos informáticos. Desde la aparición de las TIC, se debería haber comenzado a introducir en las escuelas españolas el llamado B-Learning. Este sistema se basa en la unión de la educación tradicional, cara a cara, con la educación virtual; lo que proporciona una mayor facilidad a la hora establecer el contacto profesor-alumno. Tras el estudio realizado por Santiago Alonso-García, Encarnación Morte-Toboso \& Sebastián Almansa Núñez (2015) en el que analizan el funcionamiento específico de la plataforma EDMODO para la docencia, podemos comprobar que sin la necesidad de la inversión de un gran período de tiempo en la formación para el uso de esta herramienta, la comunicación y seguimiento de la docencia una vez terminado el horario lectivo, se ve altamente fomentado por esta aplicación. Después de esta prueba, los niños hablaban muy positivamente de este medio por el cual se podían poner en contacto con el docente o podían continuar con lo que no se consigue terminar en clase. 
Las diversas plataformas que hoy día están a nuestra disposición son todo un reto para aquellos maestros que en su día no estudiaron en un mundo dirigido por las nuevas tecnologías y es por ello que en muchos casos es todo un reto continuar con la docencia a distancia.

Si ponemos el ojo en otros países como Finlandia con unos resultados académicos más elevados que los españoles nos damos cuenta de que con una limitación en los accesos universitarios en el que no sólo se valoran conocimientos generales sino la motivación y actitud para ejercer como profesores en un futuro, la formación que estos reciben puede ser más completa y personalizada. Las entrevistas y exámenes particulares de cada universidad para los futuros estudiantes se aseguran de que los educandos que están formando están ahí porque verdaderamente quieren y han dedicado su tiempo para estudiar y obtener los resultados necesarios para alcanzar esta formación.

Por otro lado, hemos considerado bastante relevante el hecho de una formación continua y una evaluación nacional del profesorado ya que es una forma de continuar con la calidad educativa que tanto les caracteriza y se considera una motivación para seguir esforzándose y formándose en los nuevos aspectos que conciernen a su formación.

La mejora que necesita nuestro país es algo que no se va a llevar a cabo de la noche a la mañana pero es por ello que debemos tener en cuenta las debilidades para conseguir realizar los cambios oportunos y necesarios. Continuar evitando las flaquezas existentes en nuestro sistema solo ocasionará la continua bajada de las medias de los expedientes españoles y el aumento del menosprecio que vive nuestra profesión.

\section{REFERENCIAS BIBLIOGRÁFICAS}

Alonso. S., y Alonso, M.M. (2014). Las redes sociales en las universidades españolas. Revista de Comunicación de la SEECI, 2014, 33, 132-140. https://www.redalyc.org/pdf/5235/523552851009.pdf

Alonso-García, S., Morte-Toboso, E., \& Almansa-Núñez, S. (2015). Redes sociales aplicadas a la educación: EDMODO. $\quad \underline{\text { http://150.214.110.170:8080/bitstream/handle/10396/13513/Edmetic }}$ vol 4 n 2 27.pdf?sequence $=1 \&$ is Allowed $=\mathrm{y}$

“Docente”. Autor: María Estela Raffino. De: Argentina. Para: Concepto.de. Disponible en:https://concepto.de/docente/\#ixzz6KeHXpuxG. Consultado: 24 de abril de 2020. 
Fernández Martín, F., Hinojo Lucena, F., \& Aznar Díaz, I. (2013). Las actitudes de los docentes hacia la formación en tecnologías de la información y comunicación (TIC) aplicadas a la educación. Contextos Educativos. Revista de Educación, O(5), 253-270. doi:https://doi.org/10.18172/con.516

García Perales, N., \& Martín Sánchez, M. A. (2012). Algunas notas en perspectiva comparada sobre formación de maestros: el caso de España y Finlandia. Tejuelo, 13(1), 70-87. Recuperado de http:// hdl.handle.net/10662/4681

Marín, V., \& Romero, M. A. (2009). La formación docente universitaria a través de las TIC. Revista de Medios y Educación, 35, 97-103. Recuperado de https://idus.us.es/bitstream/handle/11441/22601/ file 1.pdf?sequence $=1 \&$ isAllowed $=\mathrm{y}$

Ministerio de Educación y Formación Profesional. (2019). La formación inicial del profesorado en España (2019.a ed.). Madrid, España: Fondo de Cultura Económica.

Ministerio de Educación y Formación Profesional. (2019). PISA 2018. Programa para la Evaluación Internacional de los Estudiantes. Informe español. Recuperado de https://www.educacionyfp. gob.es/dam/jcr:e2be368b-f08c-4ab8-8fd9-eb93b76c6bf2/pisa-2018-programa-para-la-evaluacin-online.pdf

Niemu, H. (2015). Desarrollo profesional docente en Finlandia: Hacia un enfoque más holístico. Psychology, Sciety, \& Education, 7(3), 387-404. http://ojs.ual.es/ojs/index.php/psye/article/down$\underline{\operatorname{load} / 520 / 498}$

Orden ECI 3857/2007 de 27 de diciembre por la que se establecen los requisitos para la verificación de los títulos universitarios oficiales que habiliten para el ejercicio de la profesión de Maestro en Educación Primaria (BOE nº. 312, de 29 de diciembre de 2007).

Pérez Granados, L. (2014). La selección de candidatos a la formación docente en Finlandia. La relevancia de la disposiciones personales hacia la actividad docente. Revista electrónica de Investigación y Docencia (REID), 12, 109-132. https://revistaselectronicas.ujaen.es/index.php/reid/ article/view/1306/1510

Rebolledo Gámez, T. (2015). La formación inicial del profesorado de educación primaria y secundaria en Alemania, España, Finlandia, Francia y Reino Unido. Revista Española de Educación Comparada, 25, 129-148. https://doi.org/10.5944/reec.25.2015.14787

Rupérez, F. (2015). «MIR educativo» y profesión docente. Un enfoque integrado. Revista española de pedagogía., 261, 283-299. Recuperado de http://www.educacionyfp.gob.es/yosoyprofe/dam/ jcr:d071eaba-766d-4858-906b-2a0a753a4f2b/mir-educativo_rep_flr.pdf

Rupérez, F. (2018). Los por qués del MIR educativo. Recuperado de https://www.ucjc.edu/wp-content/ uploads/MIREDUCATIVO FLR.pdf 
Tárraga-Mínguez, R., Sanz-Cervera, P., Pastor-Cerezuela, G., \& Fernández-Andrés, M. (2017). Análisis de la autoeficacia percibida en el uso de las TIC de futuros maestros y maestras de Educación Infantil y Educación Primaria. Revista Electrónica Interuniversitaria De Formación Del Profesorado, 20(3), 107-116. https://doi.org/10.6018/reifop.20.3.263901

Zaragaza, J. M. E. (2006). Comentarios a los Informes EURYDICE y OCDE sobre la cuestión docente . Revista de Educación, 340, 19-86. Recuperado de http://www.academia.edu/download/31764438/ re340 03.pdf 